\section{Gastric liposarcoma presenting as a huge pedunculated polyp}
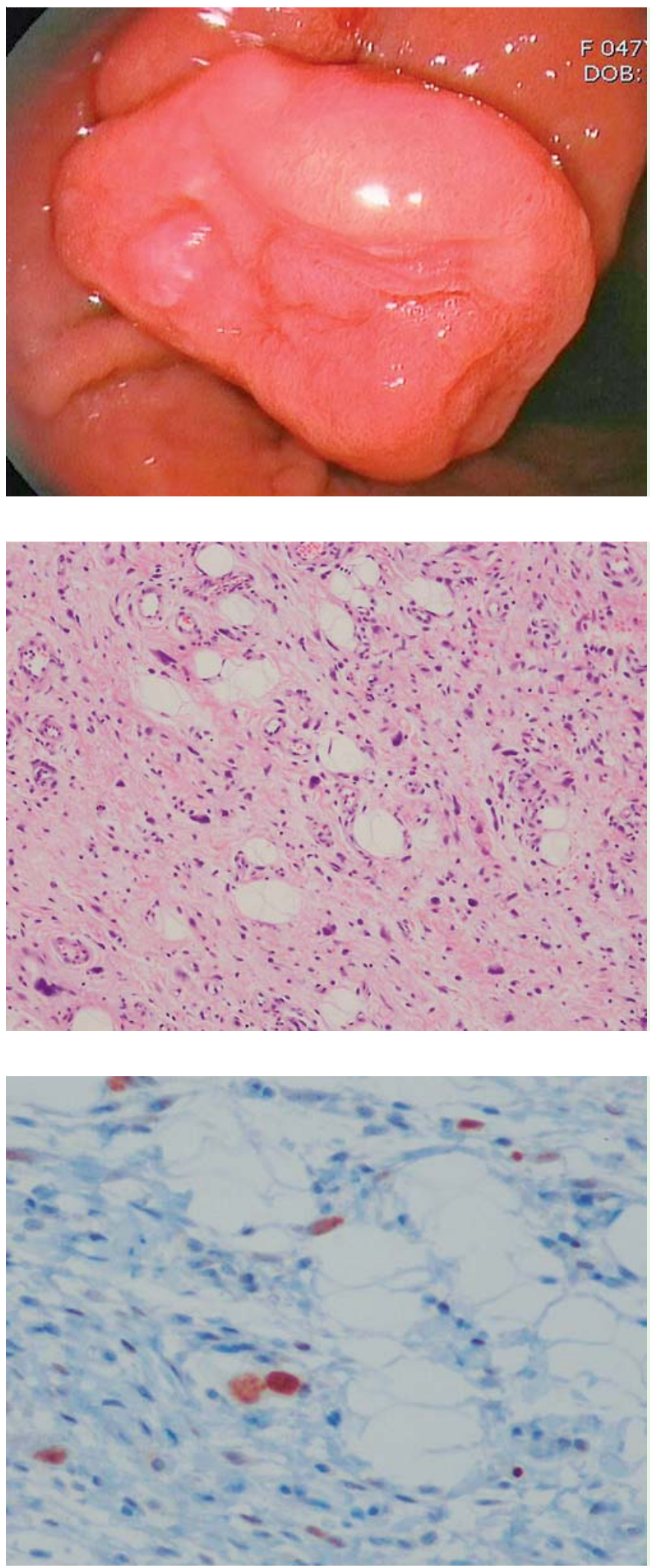

Fig. 1 Endoscopic view showing a huge pedunculated polyp.

Fig. 2 Microscopic findings showed variable-sized, mature adipocytes and scattered, bizarre, stromal cells in a fibrillary and collagenous stroma.

Fig.3 Immunohistochemical stain shows nuclear positivity of bizarre, stromal cell for MDM2.
A 46-year-old woman was admitted to a local medical center with abdominal pain and discomfort, which she had experienced for 6 months. Her medical history was unremarkable. Esophagogastroduodenoscopy (EGD) showed a huge pedunculated polyp located in the stomach body. The patient was referred to our hospital for endoscopic submucosal dissection (ESD) with the working diagnosis of hyperplastic polyp.

On repeat EGD, an 8-cm pedunculated and lobulated polyp with broad base was observed in the posterior wall of the distal gastric body ( $\bullet$ Fig. 1). Laparoscopic distal gastrectomy was performed because the polyp was too large for ESD. On gross examination, the tumor, which measured $7 \mathrm{~cm}$, was ill-defined with a fibro-fatty appearance and mixed elastic-soft and solid consistency. Microscopically, the tumor consisted of mature adipocytes, with variation in cell size and scattered, bizarre, hyperchromatic stromal cells in a fibrillary and collagenous background $(\bullet$ Fig. 2). The tumor cells showed positive nuclei staining for MDM2 ( $\bullet$ Fig.3) and CDK4 ( $\bullet$ Fig.4). The final histopathological diagnosis was that of a well-differentiated liposarcoma. The patient was considered for adjuvant treatment because the resection margin was positive for liposarcoma.

Liposarcoma accounts for $15 \%-20 \%$ of all sarcomas and usually affects the extremities and retroperitoneum [1]. However, gastric liposarcoma is extremely rare, with only 13 cases reported in the literature. The recently updated World Health Organization classification recognizes four major subtypes of liposarcoma: atypical lipomatous tumor/well-differentiated, de-differentiated, myxoid, and pleomorphic liposarcoma [2,3]. Although onethird of well-differentiated liposarcomas show local recurrence, metastasis is virtually never seen unless de-differentiation occurs [3]. The most important prognostic factor is anatomical location $[1,4]$. Overall mortality ranges from $0 \%$ for atypical lipomatous tumor of the extremities to nearly $80 \%$ for tumors occurring in the visceral sites and retroperitoneum [2,3].

Endoscopy_UCTN_Code_CCL_1AB_2AD_3AB

Competing interests: None 


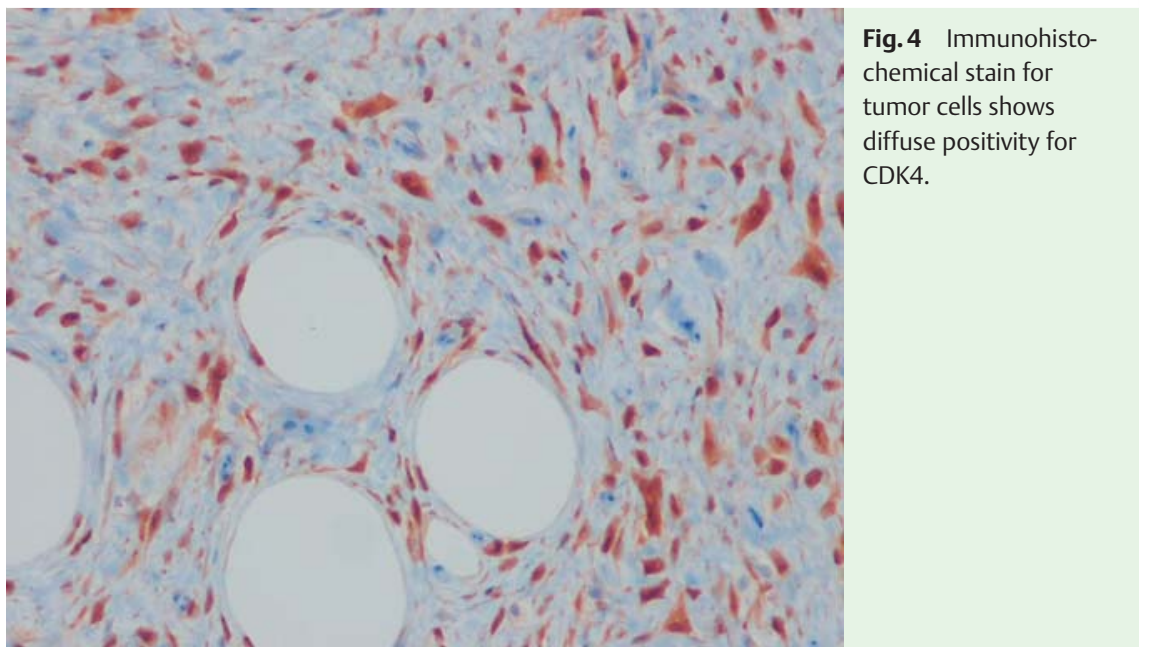

Min Jong Kim ${ }^{1}$, Mi Jin Gu ${ }^{1}$, Joon Hyuk Choi $^{1}$, Se Won Kim², Kyeong Ok Kim ${ }^{3}$

${ }^{1}$ Department of Pathology, Yeungnam University College of Medicine, Daegu, Republic of Korea

2 Department of Surgery, Yeungnam University College of Medicine, Daegu, Republic of Korea

${ }^{3}$ Department of Internal Medicine, Yeungnam University College of Medicine, Daegu, Republic of Korea

\section{Bibliography}

Dol http://dx.doi.org/

10.1055/s-0034-1377501

Endoscopy 2014; 46: E441-E442

(c) Georg Thieme Verlag KG

Stuttgart · New York

ISSN 0013-726X

\section{Corresponding author}

\section{Mi Jin Gu, MD, PhD}

Department of Pathology

Yeungnam University College of Medicine 170, Hyeonchung-ro

Nam-gu

Daegu 705-717

Republic of Korea

Fax: +82-53-6228432

mjgu@yu.ac.kr 